\title{
Erratum to: Beyond Standard Model Collider Phenomenology of Higgs Physics and Supersymmetry
}

\author{
Erratum to: \\ M.C. Thomas, Beyond Standard Model Collider \\ Phenomenology of Higgs Physics and Supersymmetry, \\ Springer Theses, DOI 10.1007/978-3-319-43452-0
}

The book was inadvertently published with incorrect abstracts in the online version and missed abstracts in the print version. The placement of citation of the equations was corrected in Chaps. 2 and 3. The erratum chapter has been updated with these changes.

\section{Chapter 1}

This introductory chapter introduces the Standard Model of particle physics, including its achievements and outstanding problems/issues. It then goes on to discuss the most promising "Beyond Standard Model" possibilities to resolve these issues, focusing on and discussing Supersymmetry and Effective Field Theories. It concludes by explaining the plan for the rest of the thesis.

DOI 10.1007/978-3-319-43452-0_1

\section{Chapter 2}

If the electroweak symmetry breaking is originated from a strongly coupled sector, as for instance in composite Higgs models, the Higgs boson couplings can deviate from their Standard Model values. In such cases, at sufficiently high energies there could occur an onset of multiple Higgs boson and longitudinally polarised electroweak gauge boson $\left(V_{L}\right)$ production. This chapter studies the sensitivity to anomalous Higgs couplings in inelastic processes with 3 and 4 particles (either Higgs bosons or $V_{L}$ 's) in the final state. It is shown that, due to the more severe cancellations in the corresponding amplitudes as compared to the usual 2 to 2

The updated original online version for this book can be found at DOI 10.1007/978-3-319-43452-0 
processes, large enhancements with respect to the Standard Model can arise even for small modifications of the Higgs couplings. In particular, triple Higgs production provides the best multiparticle channel to look for these deviations. The consequences of multiparticle production at the LHC is briefly explored.

DOI 10.1007/978-3-319-43452-0_2

\section{Chapter 3}

In this chapter, a combination of two main observables which provides a unique sensitivity to the ratio of the longitudinal versus transverse polarizations of the $\mathrm{W}$ and $\mathrm{Z}$ bosons in the vector-boson scattering processes is introduced. It is shown that these observables allow sensitivity to the Higgs boson couplings to the gauge bosons and consequently to the theory underlying the Higgs sector. We conclude that the analysis of vector boson fusion provides a model independent and robust method to study the Higgs boson couplings to the gauge bosons.

DOI 10.1007/978-3-319-43452-0_3

\section{Chapter 4}

In this chapter, the effects from light sfermions on the lightest Higgs boson production and decay at the Large Hadron Collider (LHC) within the Minimal Supersymmetric Standard Model (MSSM) is studied. It is found that the scenario with light coloured sfermions (stops or sbottoms) has the potential to explain a non-universal alteration, as hinted by LHC data, of the gluon-gluon Fusion $\left(\mu_{g g F}\right)$ with respect to the Vector Boson Fusion (VBF) event rates and, in particular, can predict $\mu_{V B F} / \mu_{g g F}>1$ for all Higgs boson decay channels in large areas of the parameter space. The scenario with a light stop is emphasised, as the latter is also motivated by Dark Matter and Electro-Weak baryogenesis, although we also explore scenarios with light sbottoms and/or staus as well as a SUSY induced reduced bottom Yukawa. Fits of the MSSM against the LHC data is performed, emphasising the fact that in most cases these are better than for the SM.

DOI 10.1007/978-3-319-43452-0_4

\section{Chapter 5}

Understanding the extent to which experimental searches are sensitive to scenarios involving Light Stops (LST) is essential to resolve questions about naturalness, electroweak baryo-genesis and Dark Matter. In this chapter, the reach on LST scenarios is characterised in two ways. Firstly, we extend experimental searches to cover specific gaps in the LST parameter space, showing for the first time that assuming a single decay channel one can exclude the region of $m_{\tilde{t}}<m_{\text {top }}$, which in its turn excludes electroweak baryogenesis in MSSM. Secondly, we explore the extent to which searches are weakened in a more generic scenario when more than one decay channel takes place, even after their combination. This work highlights the need for a more comprehensive exploration of the LST parameter space.

DOI 10.1007/978-3-319-43452-0_5 


\section{Chapter 6}

This concluding chapter summarises and discusses the results of this thesis, and their current and future implications for searches at the Large Hadron Collider.

DOI 10.1007/978-3-319-43452-0_6

In Chap. 2 citation has been removed from Eq. 2.20 and added it to the last line of text before the equation.

In addition, we produced a set of events with additional VBF cuts [29]:

$$
\text { VBF cuts: } E_{j}>300 \mathrm{GeV}
$$

In Chap. 3 citation has been removed from Eq. 3.5 and added it to the last line of text before the equation.

In this case we used the following kinematic cuts ${ }^{3}[18]$ :

$$
\text { VBF cuts: } \Delta \eta_{j j}>4, E_{j}>300 \mathrm{GeV}
$$

